\title{
A SECURED HEURISTIC ROUTING ALGORITHM FOR VEHICLE AD-HOC NETWORK
}

\author{
Suchandan Ganguly ${ }^{1}$, Somsubhra Gupta ${ }^{2}$ and Soumyabrata Saha ${ }^{3}$
}

\begin{abstract}
This work presents how to incorporate intelligence in designing a routing algorithm to determine nodes of the wireless sensor network (WSN) to route the packets between different nodes. In the model formulation of the problem a heuristic function has been introduced in the proposed routing algorithm to evaluate the costing of transmission to the next layer of nodes and thus selecting the receiving nodes with a cost optimized way in the next layer of transmission. By that process, the exhaustive overhead of sending data packet to each and every neighboring node can be avoided. Instead, the data packet will be send to the node with minimum heuristic value since cost optimization is the aim. In spite of intrinsic uncertainties in a dynamic framework of Vehicle Ad-Hoc Network with movable nodes under WSN framework, the use of heuristic may suitably ensure the identification of destination so as to route the information. Moreover, introduction of a linear heuristic will try to obtain a shortest path to the extent possible among the identifiable neighbour nodes in the next layer. Hence in the model formulation of the problem, a linear heuristic has been incorporated in which genetic algorithm has been employed in the solution search process.

Primarily, the system will calculate the estimated time for the message to be reached from source to destination. Furthermore, it will also perform an encryption operation on the message to be sent at the sender site and the decryption operation on the same message at the receiver site. The concept model is accompanied by a case studies developed using $\mathrm{C}$ language that incorporates the heuristic, network and the security.

Keywords - Genetic Algorithm, Heuristic, Routing, VANET, Wireless networking.
\end{abstract}

\section{INTRODUCTION}

A wireless ad hoc network (WANET) is a decentralized type of wireless network. It is called as ad hoc because it does not rely on a pre-existing infrastructure, such as routers in wired networks or access points in managed wireless networks. In this network, each node participates in routing by forwarding data for other nodes, so the determination of which nodes forward data is made dynamically on the basis of network connectivity. Ad hoc networks also use flooding for forwarding data in addition with the classic routing ${ }^{[1]}$.

There are many different types of Ad-hoc network presents. Some of them are briefly discussed here.

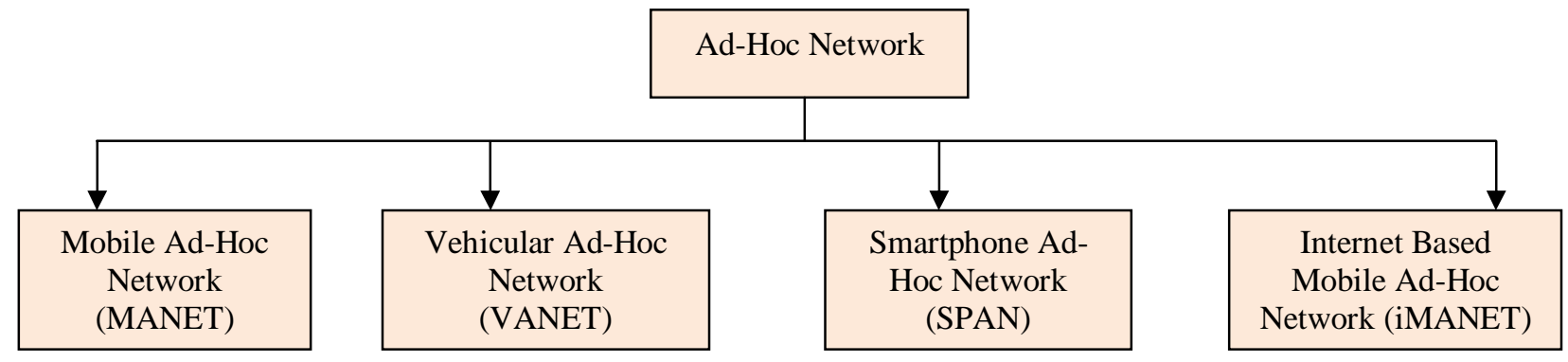

Figure 1.1: Type of Ad-Hoc network

\footnotetext{
${ }^{1}$ Department of Information Technology JIS College of Engineering, West Bengal, INDIA

${ }^{2}$ Department of Computer Science and Engineering and Information Technology JIS College of Engineering, West Bengal, INDIA

${ }^{3}$ Department of Information Technology JIS College of Engineering, West Bengal, INDIA
} 


\section{A. MANET}

A Mobile Ad-hoc Network is a collection of independent mobile nodes that can communicate with each other via radio waves. The mobile nodes that are in radio range of each other can directly communicate, whereas others need the help of intermediate nodes to route their packets. Each of the nodes has a separate wireless interface to communicate with each other. These networks are fully distributed, and can work at any place without the help of any fixed infrastructure such as access points or base stations [2].

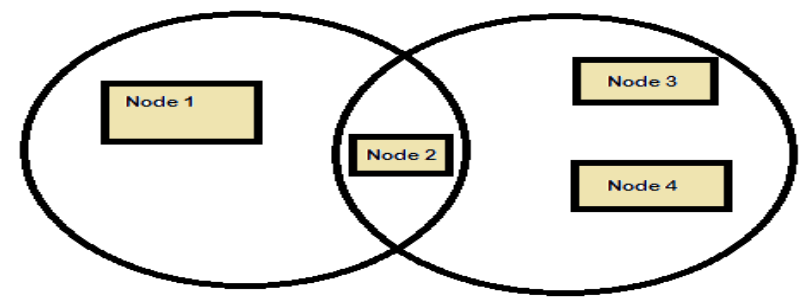

Figure 1.2: Mobile Ad hoc Network

\section{B. VANET}

- Vehicular ad hoc network, consisting of a network of vehicles, moving at a relatively high speed, that communicate among themselves with different purposes, being the main purpose that of improving security on the road.

- Vehicular Ad Hoc Networks (VANETs) are created by applying the principles of mobile ad hoc networks (MANETs) - the spontaneous creation of a wireless network for data exchange - to the domain of vehicles.

- Vehicular Ad Hoc Networks. When principles of MANET are applied in domain of vehicles, they form VANETs. A generic term used to define VANETs is inter-vehicle communication (IVC). The vehicles are fitted with sensors. These sensors interact with the sensors of other vehicles or the infrastructure present outside.

- A form of mobile ad hoc network, to provide communications among nearby vehicles and between vehicles and nearby fixed equipment, usually described as roadside equipment.

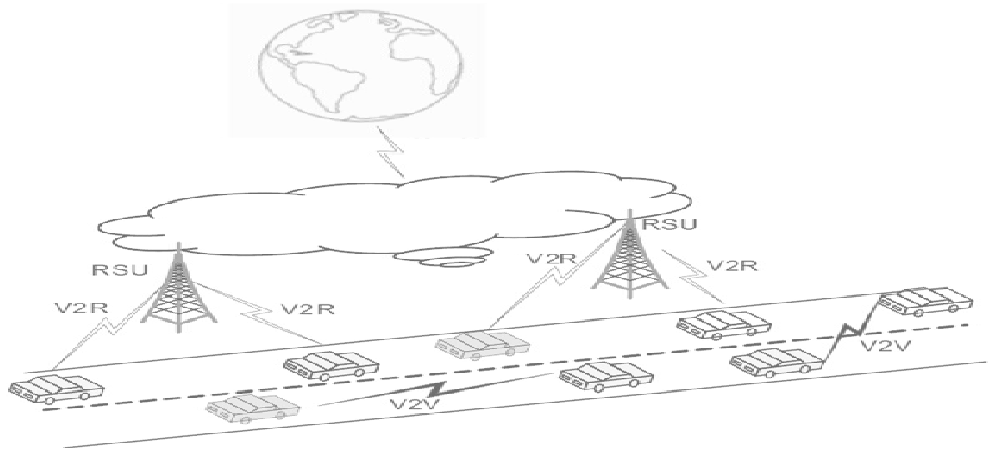

Figure 1.3: VANET Architecture

\section{SPAN}

Smart Phone Ad hoc Networks (SPANs) uses the existing hardware i.e. primarily Bluetooth and Wi-Fi available in commercial smart phones to create peer-to-peer networks without relying on cellular carrier networks, wireless access points, or traditional network infrastructure. SPANs differ from traditional hub and spoke networks, such as Wi-Fi Direct, in that they support multi-hop relays and there is no option of a group leader so peers can join and leave at will without destroying the network ${ }^{[3]}$.

\section{IMANET}

Internet-based mobile ad hoc networking is an emerging technology that supports self-organizing, mobile networking infrastructures. The technology enables an autonomous system of mobile nodes, which can operate in isolation or be connected to the greater Internet. 


\section{VANET FRAMEWORK}

\section{A. Types of Communication in VANET}

The goal of VANET architecture is to allow the communication among nearby vehicles and between vehicles and fixed roadside equipments leading to the three possibilities: V2V, V2I, and Hybrid Communication

\section{Vehicle to vehicle communication $(\mathrm{V} 2 \mathrm{~V})$}

Vehicle-to-Vehicle (V2V) ad hoc network allows the direct vehicular communication without relying on a fixed infrastructure support and can be mainly employed for safety, security, and dissemination applications [4].

Figure 2.1: Vehicle to Vehicle

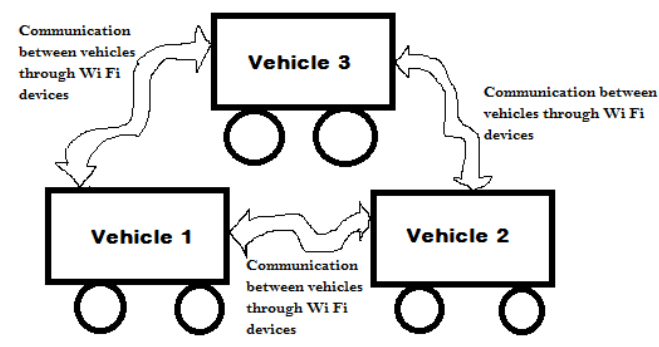

\section{Vehicle to infrastructure communication (V2I)}

Vehicle-to-Infrastructure (V2I) network allows a vehicle to communicate with the roadside infrastructure mainly for information and data gathering applications [4].

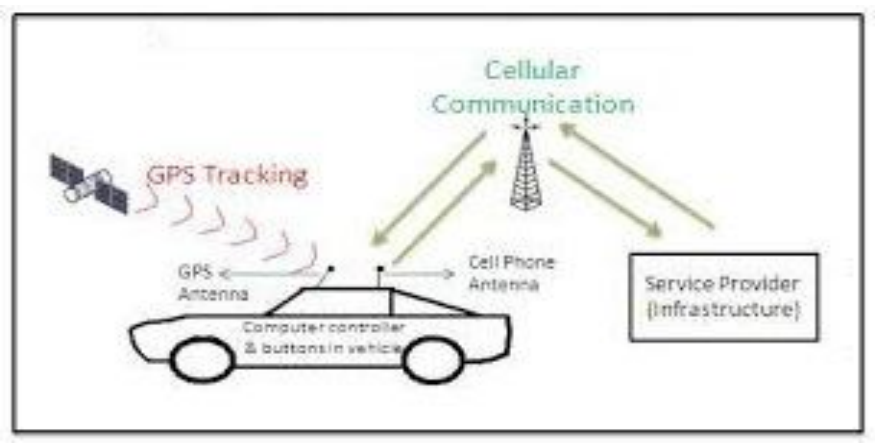

Figure 2.2: Vehicle to Infrastructure (V2I) Communication

\section{Hybrid communication}

Hybrid architecture: combines both Vehicle-to-Vehicle (V2V) and Vehicle-to-Infrastructure (V2I). In this scenario, a vehicle can communicate with the roadside infrastructure either in a single hop or multi-hop fashion, depending on the distance, i.e., if it can or not access directly the roadside unit (RSU). It enables long distance connection to the Internet or to vehicles that are far away [4].

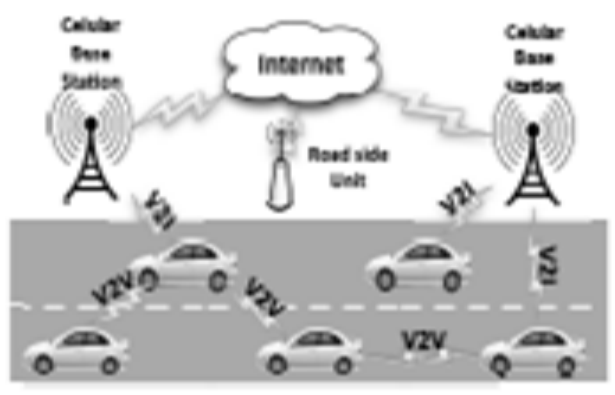

Figure 2.3: Hybrid Communication 


\section{B. VANET Problem Charasteristics}

- Security problem: The security of message content is a big issue in vehicle-to-vehicle (V2V) communication. As all the nodes in VANET are moving at a high speed, so the content of the message received is to be verified within that amount of time [5].

- Authentication problem: The authentication is a very vital issue in any type of network communication. It has to be pretty sure that the communication in between the nodes as well as in between nodes and other units has to be authentic. For that the communicating vehicles should only accept the data from authentic vehicles only [12].

- Integrity: Another major problem of VANET and any other network is integrity. Generally integrity deals with the stability of the data which is to be sent or received. It looks after that the data which is sent has received intact as it is sent i.e. it checks that any type of modification, insertion or reordering of data is happened or not [5].

- Confidentiality: It guarantees that the message which is sending can be prevent from outside unauthorized access. So this normally provides the confidentiality required for the message [5].

- Accessibility: It is another issue in any network including VANET that there must be a access permission to particular messages. Even a robust communication channel can still suffer some attacks which can bring down the network. An important feature of VANET security is the digital signature [6, 5].

- Scalability: It means that the existing network can be increased without affecting the existing system to a much extend. That is if we want to increase the number of nodes then there must be the way so that the number of nodes can be increased without affecting the existing nodes in that network or even keeping the organization of the node as it was [5].

- Reliability: Due to the brief communication time, it is difficult to assure the reliable message reception and acknowledgement between communication vehicles on opposite directions. In vehicular ad hoc networks a majority of the messages that are transmitted will be periodic broadcast messages that announce the state of a vehicle to its neighbors. So in case of broadcast messages it needs more reliability $[6,5]$.

\section{Features of VANET}

Although VANET is the application of MANET, but in VANET it has some important features. Some of them are briefly discussed below.

(i) Highly mobile: The nodes in VANET generally moves at high speed for that it makes harder to predict a node's position and it is also difficult to protect the node privacy whose position is rapidly changing [7].

(ii) Network topology: The network topology changes frequently in VANET because the nodes in the VANET are mobile and also they change their positions at a very high speed [7].

(iii) Unlimited size of network: As the nodes of VANET are vehicles, so it can be implemented within a city or within more than one city or even in a country also. So the size of the network is limitless [7].

(iv)Regular exchange of information: As VANET is a kind of ad hoc network so the nodes have to gather information from the other vehicles and road side units. Hence the information exchange among node becomes frequent [7].

(v) Time: The information in VANET must be delivered to the nodes with in time limit so that a decision can be made by the node and perform action accordingly [7].

\section{Contemporary Routing Approaches}

In VANET the routing protocols are classified into different categories, under which there are different routing algorithms. Here I had try to give a brief idea of different types of existing routing algorithms used in VANET.

- $\quad \boldsymbol{F S R}$ : FSR stands for "Fisheye state routing". There are topology tables in each nodes of FSR where the current information received from the neighboring nodes is stored. This information i.e. the routing table is exchanged with the neighboring nodes exist in the surroundings. Generally FSR uses different exchange time for different entries in the routing table in case of large network. The entries in the routing table for a given destination are updated with the help of the neighbors which have low frequency [8]. 
- DSDV: DSDV stands for "Destination sequenced distance vector routing". It is a table driven routing protocol. It is based on Bellman- Ford algorithm. It reduces the overhead of control message, increases the convergence speed and also eliminates route looping. Each and every nodes of DSDV maintains a next-hop table which it exchange with its neighbor nodes [8].

- $\quad$ OLSR : OLSR stands for "Optimized link state routing protocol". In this routing protocol the nodes select a set of neighboring nodes which is termed as multipoint relays which is responsible to transmit packets. So the nodes which are not in multipoint relays cannot able to transmit data packets they can only read and process the packet. Using this type of technique reduces the number of retransmission in the broadcast network [8].

- CGSR: CGSR stands for "Cluster head gateway switch routing". This protocol is slightly different from the other protocols in the area of addressing and network organization. It is a clustered multihop wireless network which comprises of several heuristic schemes. With the help of cluster head control a framework for code separation, allocation of bandwidth and channel access can be achieved along with routing [8].

- WRP: WRP stands for The "Wireless routing protocol". The main goal of WRP is to maintain routing information among all the nodes. It is also a table based protocol. All the nodes in the network had to maintain four tables. They are: distance table, message retransmission list, routing table and link cost table [8].

- TBRPF: TBRPF stands for "Topology dissemination based on reverse path forwarding". It is a link state routing protocol which is basically designed for ad-hoc networks.

- In this topology a source tree is constructed by all the nodes present in the network which contains paths to all the other reachable nodes by using the topology table. The nodes are updated regularly using the difference between the previous and the current network state using HELLO message [8].

- AODV: AODV stands for "Ad hoc on demand distance vector". In AODV when the node received a broadcast query (RREQ), it saves the address of the RREQ sending node in the routing table. The procedure of recording the information of previous node is called backward learning. Upon arriving at the destination a reply packet (RREP) is sent through the path which is obtained from the backward learning back to the source [8].

- PGB: PGB stands for "Preferred group broadcasting". It relies on broadcasting mechanism which try to reduce the broadcast overhead arises in AODV's route discovery. It also tries to provide route stability which is a very important thing in VANET as the nodes in VANET are vehicles which frequently changes their positions. Based on the received signal of the broadcast, receivers can determine whether they are in the preferred group and which one in the group to broadcast [8].

- DSR:DSR stands for "Dynamic source routing". It uses source routing, that is the source indicates in a data packet's the sequence of intermediate nodes on the routing path. In DSR, the query packet copies in its header the IDs of the intermediate nodes that it has traversed. The destination then retrieves the entire path from query packet which is used to respond back to the source [8].

- TORA:TORA stands for "Temporally ordered routing algorithm". It is a link reversal routing algorithm. Here a directed acyclic graph has to be built based on the height of the tree rooted at the source and whose direction will be towards the destination. The directed acyclic graph directs the flow of packets and ensures reach ability to all nodes. When a node has a packet to send, it broadcasts the packet. Its neighbor only broadcasts the packet if it is the sending node's downward link based on the directed acyclic graph [8].

- ZRP: ZRP stands for "zone routing protocol". In this the network is divided into overlapping zones. The zone is defined as a collection of nodes which are in a zone radius. The size of a zone is determined by a radius of length $\alpha$ where $\alpha$ is the number of hops to the perimeter of the zone. In ZRP, a proactive routing protocol (IARP) is used in intra-zone communication and an inner-zone reactive routing protocol (IARP) is used in intrazone communication. Source sends data directly to the destination if both are in same routing zone [8].

- HARP:HARP stands for "Hybrid ad hoc routing protocol". It divides entire network into non-overlapping zones. It aims to establish a stable route from a source to a destination to improve delay. It applies route discovery between zones to limit flooding in the network, and choose best route based on the stability criteria. In this routing protocol, routing is performed on two levels: intra-zone and inter-zone, depending on the position of destination. It uses proactive protocol in intra-zone routing and reactive protocols in inter-zone routing [8].

- GPSR: GPSR stands for "Greedy perimeter stateless routing". In this routing protocol a node forwards a packet to an immediate neighbor which is geographically closer to the destination node. This mode of forwarding is termed greedy mode. When a packet reaches a local maximum, a recovery mode is used to forward a packet to a node that is closer to the destination [8].

- GPCR: GPCR stands for "Greedy perimeter coordinator routing". It is a geographic routing algorithm that utilizes the fact that the nodes at a junction in the street follow a natural planar graph. Thus, a restricted greedy algorithm can be followed as long as the nodes are in a street, and junctions are the only places where actual 
routing decisions are taken. Therefore packets should always be forwarded to a node on a junction rather than being forwarded across the junction. Apart from the greedy routing strategy, when GPCR encounters a local optimum, it uses a repair strategy to get out of it. The repair strategy decides, on each junction, which street the packet should follow next using the right-hand rule [9].

- GSR: GSR stands for "Geographic source routing". It is a position-based routing protocol with topological information. This routing protocol uses street map in order to acquire a global knowledge of the city topology and a Reactive Location Service (RLS) to get the destination position. With the help of this information, the algorithm first determines the junctions that have to be traversed by the packets and then applies greedy forwarding between them [9].

- A-STAR : A-STAR stands for "Anchor-based street and traffic aware routing". This routing algorithm uses information on city bus routes to identify an anchor path with high connectivity for packet delivery. By using an anchor path, it guarantees to find an end-to-end connection even if traffic density is low. This algorithm also employs a route recovery strategy when the packets are routed to a local optimum by computing a new anchor path from the local maximum to which the packet is routed [9].

- GyTAR : GyTAR stands for "Greedy traffic aware routing protocol". It is an intersection-based geographical routing protocol capable of finding robust routes within city environments. To select appropriate paths this routing protocol requires the existence of an accurate traffic information system. It takes into account the position of the junctions in order to decide the next hop for each packet [9].

- DIR: DIR stands for "Diagonal intersection based routing protocol". The DIR protocol is based upon the geographic routing protocol. This protocol built a series of diagonal intersections between the source and destination vehicle in which source vehicle geographically forwards the data packets towards the first diagonal intersection then second diagonal intersection and so on until the last diagonal intersection and finally geographically reaches to designation or goal vehicle [8].

- CAR: CAR stands for "Connectivity aware routing protocol". It is a combination of characteristics from both geographic routing protocols, like GPSR [8], and ad-hoc routing protocols, like AODV [8]. It consists of 4 main phases: path discovery, data forwarding, with the help of guards and error recovery. However from the simulation results it is clear that this routing protocol is performance wise good but it is very complex protocol due to of many phases it has [9].

- $\quad \boldsymbol{C B F}: \mathrm{CBF}$ stands for “Contention based forwarding”. It is a geographic routing protocol. It doesn't make use of beacons. Here if there is a data packet to send, the sending node will broadcast the packet to all direct neighbors. These neighbors in turn will find out among themselves the one that will forward the packet. As this technology doesn't use beacon message so it saves the bandwidth [8].

- VADD: VADD stands for "Vehicle assisted data delivery". VADD is a vehicular routing strategy whose aim is to improve routing in disconnected vehicular networks. To achieve this it uses carry and forward based on the use of predictable vehicle mobility. A vehicle simply makes a decision at a junction and selects the next forwarding path with the smallest packet delivery delay. A path is nothing but simply a branched road from an intersection [9].

- $\quad \boldsymbol{G e o D T N}+\boldsymbol{N A V}:$ GeoDTN + NAV stands for "Geographic DTN routing with navigator prediction" [8]. It is a hybrid of non-DTN and DTN approach. It is a combination of greedy mode, the perimeter mode, and the DTN mode. It switches from non-DTN mode to DTN mode by estimating the connectivity of the network based on the number of hops a packet has travelled so far, neighbor's delivery quality, and neighbor's direction with respect to the destination [9].

- $\quad \boldsymbol{C B D R P}$ : CBDRP stands for "Cluster-based directional routing protocol". It divides the vehicles into clusters. Here the vehicles which are moving in same direction from a cluster. The source sends the message to its cluster header and then the cluster header in turn forwards the message to header which is in the same cluster with the destination. At last the destination header sends the message to the destination. During the selection of the cluster header both the velocity and the direction of the vehicle are taken into consideration [8].

- $\quad$ LORA- CBF : LORA-CBF stands for "Location routing algorithm with cluster based flooding". In this routing algorithm each node can become the cluster-head, gateway or cluster member. There is only one cluster head for each cluster. The node which connects two clusters are called gateway.

- The cluster head maintains information about its members and gateways. The packet forwarding is same as in the greedy routing. Only cluster head and gateways can send out the location request (LREQ) packets when the location of the destination is not available [8].

- $\quad \boldsymbol{H C B}$ : HCB stands for "Hierarchical cluster based routing". It is a hierarchical cluster routing protocol designed for highly movable ad hoc networks. This routing algorithm comprises of two layer communication architecture. In layer-1 most of the nodes have single radio interface and they can communicate via each other 
through multi hop path. Among these nodes some also have another interface with long radio communication range called super nodes.In layer-2 super nodes are able to communicate with each other via the base station. Super nodes are exists both in layer-1 and in layer-2 [8].

- CBLR: CBLR stands for "Cluster based location routing". This algorithm assumes that all the vehicles can able to gather their positions via GPS (Global Positioning System). The algorithm divides the network into multiple clusters. Each cluster consists of a cluster-head and a group of members within the transmission range of the cluster-head. The rule behind the formation of cluster-head and members are as follows: When a new vehicle transmits a "Hello Message", if the vehicle gets a reply from the cluster-head vehicle, the new vehicle would become a member of the cluster. If not, the new vehicle becomes the cluster head [8].

- $\quad \boldsymbol{C B R}$ : CBR stands for "Cluster Based routing protocol". Its routing protocol is based on position and clusters. In this routing protocol, the geographic area is divided into some foursquare grids. Only if there is a vehicle in a grid then that vehicle will be elected to be the cluster header, and the data packet is routed by cluster header across some grids one by one [8].

- IVG: IVG stands for "Inter vehicle geocast". It was developed especially for transmitting safety messages to vehicles on a highway. The protocol uses a timer based mechanism for message forwarding and periodic broadcasts are used to overcome network fragmentation [8].

- DRG: DRG stands for "Distributed robust geocast". This routing protocol improves the reliability of message forwarding by defining the zone of forwarding (ZOF) which surrounds the region of interest. Vehicles in the zone of forwarding region forward the message to other vehicles in the region of interest [8].

- UMB: UMB stands for "Urban multihop broadcast protocol". Problems like packet collision, interference and hidden node arises during message transmission in multihop broadcast, these problems can be overcome with the help of UMB protocol. In UMB the sender node tries to select the furthest node in the broadcast direction for forwarding and acknowledging the packet. There is no topology information present at the beginning of data transmission. The UMB protocol has much success where the traffic load and packet density is much higher [8].

- DV-CAST: DV-CAST stands for "Distributed vehicular broadcast protocol". For broadcasting the information it uses local topology information by using the periodic hello messages. Each vehicle uses a flag variable to check whether the packet is redundant or not. This protocol divides the vehicles into three types depending on the local connectivity. The division are well connected, sparsely connected and totally disconnected [8].

- SRB: SRB stands for "Secure ring broadcasting". SRB protocol is used to minimize number of retransmission messages and to get more stable routes. It classifies nodes into three groups based on their receiving power. They are- Inner Nodes (close to sending node), Outer Nodes (far away from sending node) and Secure Ring Nodes (preferable distance from sending node). Rebroadcasting of messages is only done to secure ring nodes because it will minimize number of retransmissions [8].

\section{Methodological Aspects}

\section{A. Heuristic approach}

As the whole piece of work is based on ad-hoc network so there must be uncertainty. To transmit data to the next node will be little bit of difficult as the choice of next node is not very easy. At a certain time a specific node can be detected to transmit data but at the time data transmission it may be observed that that node is not found at its intended position because the nodes in the ad-hoc network are moving in nature.

So, a mechanism is required so that a better path from source to destination can be achieved which will also be minimum covered distance between source to destination. In my project I had calculated the distance from the longitude and latitude of the destination with other nodes. According to the minimum distance the data is transmitted to that node. As the data transmitted to the node is of the minimum distance and all the transmitting node will be selected based on their minimum distance with the destination, so the data transmitting path will guarantee that it will the minimum path among all others path present.

The whole project is implemented in c language because to make it more comfortable to understand and to make it very simple.

\section{B. Distance Calculation from latitude and longitude}

Here I use the 'haversine' formula to calculate the great-circle distance between two points - that is, the shortest distance over the earth's surface [10]. 
dlon=lon2-lon 1

dlat=lat2-lat 1

Where, lon $1 \&$ lat 1 are the longitude \& latitude of the respective node lon $2 \&$ lat 2 are the longitude \& latitude of the destination node

$$
\begin{aligned}
& a=\left(\sin (\mathrm{dlat} / 2)^{2}+\cos (\text { lat } 1) * \cos (\text { lat } 2) *(\sin (\mathrm{dlon} / 2))^{2}\right. \\
& \mathrm{c}=2 * \mathrm{a}^{*} \tan 2(\operatorname{sqrt}(\mathrm{a}), \operatorname{sqrt}(1-\mathrm{a})) \\
& \mathrm{dN}_{\mathrm{i}}=\mathrm{R}^{*} \mathrm{c}
\end{aligned}
$$

In which $\mathrm{R}=$ radius of the Earth i.e. $6,371 \mathrm{Km}$ or 3,961 miles [11].

$\mathrm{c}$ is the angular distance in radians, and a is the square of half the chord length between the points.

Using atan2 () function I had calculated the arc tangent in radians of sqrt(a)/sqrt(1-a) based on the signs of both values to determine the correct quadrant.

\section{Arctangent}

The arctangent of $\mathrm{x}$ is defined as the inverse tangent function of $\mathrm{x}$ when $\mathrm{x}$ is real.

When the tangent of $\mathrm{y}$ is equal to $\mathrm{x}[20]$ :

$$
\tan y=x
$$

Then the arctangent of $\mathrm{x}$ is equal to the inverse tangent function of $\mathrm{x}$, which is equal to $\mathrm{y}$.

For Example:-

$$
\text { i.e. } \arctan x=\tan ^{-1} x=y
$$

$$
\arctan 1=\tan ^{-1} 1=\Pi / 4 \mathrm{rad}=45^{\circ}
$$

Generally arctangent i.e. atan or $\tan ^{-1}$ is used to calculate the angles of a right triangle. It works opposite of the tangent function. While tangent will find the ratio of the two sides of a right triangle when given an angle, arctangent can find the angle given the ratio [12].

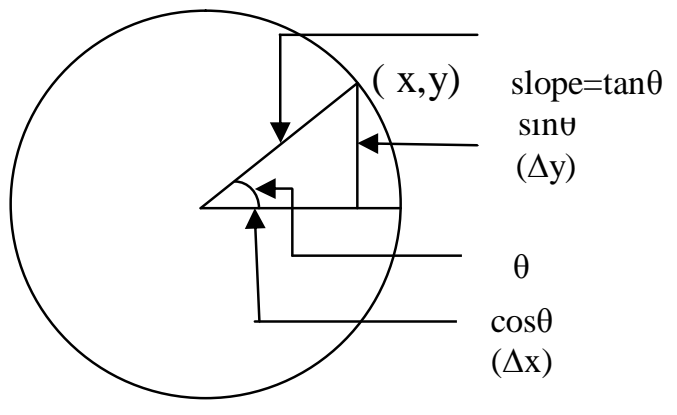

$\tan \theta=\sin \theta / \cos \theta$

Fig 4.1: Concept of arctangent

The concept of Genetic algorithm in extension to the incorporation of Heuristic has been presented in the following subsection:

\section{Proposed Algorithms}

The algorithms are presented sequentially in the following Section

A. Proposed Heuristic Routing Algorithmon VANET

Algorithm 1:

Let a vehicle i.e. a node $\mathrm{N}_{\mathrm{i}}$ will send the data packet to be transmitted to its registered RSU.

begin:

Step-1: RSU will check that if any other registered Ni of that RSU is goal node or not.

Step-2: if $\left(\mathrm{N}_{\mathrm{i}}==\right.$ goal node $)$

Step-2.1: Deliver the data packet to node Ni and quit the process. 
Step-3: For $\mathrm{i}=1$ to $\mathrm{RN}_{\mathrm{i}}$ do

Step-3.1: Compute heuristic function $\mathrm{dN}_{\mathrm{i}}$ by

$$
\mathrm{dN}_{\mathrm{i}}=\left(\mathrm{LLDN}_{-}-\mathrm{LN}_{\mathrm{i}}\right) \text { [Algorithm 1.a] }
$$

Step-3.2: Return smallest $\mathrm{dN}_{\mathrm{i}}$ which will be denoted as $\operatorname{sdN}_{\mathrm{i}}$ [Algorithm 1.b]

Step-4: RSU will sent data packet to the node $\left(\mathrm{N}_{\mathrm{i}}\right)$ with value $\operatorname{sdN}_{\mathrm{i}}$

Step-5: The node $\left(\mathrm{N}_{\mathrm{i}}\right)$ search the location of its neighboring node

Step-6: if $\left(\mathrm{N}_{\mathrm{i}}==\right.$ goal node $)$

Step-6.1: Deliver the data packet to the node $\left(\mathrm{N}_{\mathrm{i}}\right)$ and quit the process

Step-7: The node Ni will compute $\mathrm{dNi}$

$$
\text { For } \mathrm{i}=1 \text { to } \mathrm{sN}_{\mathrm{i}}
$$

Step-7.1: Compute heuristic function by

$$
\mathrm{dN}_{\mathrm{i}}=\left(\mathrm{LLDN}-\mathrm{LLsN}_{\mathrm{i}}\right) \text { [Algorithm 1.a] }
$$

Step-7.2: Return smallest dNi i.e. $\operatorname{sdN}_{\mathrm{i}}$ [Algorithm 1.b]

Step-8: The node Ni will transmit the data packet to node $\mathrm{sN}_{\mathrm{i}}$ which has the smallest value of $\mathrm{sdN}_{\mathrm{i}}$ Step-9: The node with smallest value $\operatorname{sdN}_{\mathrm{i}}$ will transmit the packet to its registered RSU.

Step-10: Repeat from Step-1 until goal node is found

end

The meaning of the symbols are as follows:

RSU- Road Side Unit

$\mathrm{RN}_{\mathrm{i}}-$ Number of Registered Node

$\mathrm{dN}_{\mathrm{i}}-$ Distance of node and the destination node

$\mathrm{sdN}_{\mathrm{i}}-$ Smallest distance Node

$\mathrm{sN}_{\mathrm{i}}-$ Searched Node

The algorithm written in the following section will compute the distance of the nodes taking into consideration the latitude and longitude of it.

\section{B. Distance Calculaton from latitude and longitude}

\section{Algorithm 2:}

Read the value of latitude and longitude of source node as lon 1 and lat1 respectively and the latitude and longitude of destination node as lon2 and lat2 respectively.

begin:

Step 1: Compute the value of dlon and dlat as follows:

$$
\begin{aligned}
& \text { dlon= lon2-lon } 1 \\
& \text { dlat=lat2-lat } 1
\end{aligned}
$$

Step 2: Compute the value of dlatmod and dlonmod as follows:

dlatmod $=(\mathrm{dlat} / 2)$

dlonmod $=($ dlon/2)

Step 3: Compute ' $a$ ' which is the square of half the chord length between the Source and the destination. $\mathrm{a}=(\sin ($ dlatmod $) * \sin ($ dlatmod $))+(\cos ($ lat 1$) * \cos ($ lat 2$) *(\sin ($ dlonmod $) * \sin ($ dlonmod $)))$

Step 4: Compute ' $c$ ' which is the angular distance in radians between the source and the destination.

$$
\mathrm{c}=2 *(\operatorname{atan} 2(\operatorname{sqrt}(\mathrm{a}), \operatorname{sqrt}(1-\mathrm{a})))
$$

Step 5: Now compute 'd' the distance between source and destination as follows:

$$
\mathrm{d}=\mathrm{R}^{*} \mathrm{c}
$$

Where $\mathrm{R}$ is the radius of the Earth i.e. 6,371 Km or 3,961 miles.

end

After obtaining the distance from the above algorithm the algorithm written in the section-7.3 will sort the distances in ascending order and thus will return the smallest distance among them.

\section{Calculation of Shortest distance}

\section{Algorithm 3:}

All the distances which are computed by Algorithm 1.a are stored into an integer array. Now read that array i.e. a[] here and the total number of nodes i.e. $\mathrm{k}$ here. 
begin:

Step-1: For $\mathrm{i}=\mathrm{k}$ to 0

Step-1.1: Compute the value of 'small' and 'pos' as follows:

$$
\text { small }=\mathrm{a}[0]
$$

pos $=0$

Step- 1.2: For $\mathrm{j}=1$ to $\mathrm{i}$

Step-1.2.1: if(a[j] > small)

Step-1.2.1.1: small $=\mathrm{a}[\mathrm{j}]$

Step-1.2.1.2: pos $=\mathrm{j}$

Step-1.2.2: End of if

Step-1.3: End of For

Step-1.4: a[pos] $=\mathrm{a}[\mathrm{i}]$

Step-1.5: a[i] =small

Step-2: End of For

end

The algorithm written in the section-7.4 will perform both the encryption and the decryption of the message sent.

\section{Cyphering-decyphering message}

The message which is to be encrypted is stored into a char array named as data[]. Actually this is a function which is written in a '.h' file and is included in the main program.

\section{Algorithm 4:}

begin:

Step 1: Read the message to be encrypted.

Step 2: Declare the variables enkey $=1 \&$ dckey $=1$.

Step 3: For i=0 to Null(' 10 ') do

Step-3.1: Compute endata[j] by, endata $[j]=$ data $[\mathrm{i}]+$ enkey +10

Step-3.2: Increment the value of ' $\mathrm{j}$ '.

Step-3.3: Increment the value of 'enkey'.

Step 4: Intialize endata[j] $=' 10$ '.

Step 5: Print the value of encrypted message.

Step 6: For i=0 to Null(' 10 ') do

Step 6.1: Compute dcdata[k] by, dcdata $[\mathrm{k}]=$ endata[i]-dckey-10

Step 6.2: Increment the value of ' $\mathrm{k}$ '.

Step 6.3: Increment the value of 'dckey'.

Step-7: Print the value of decrypted message.

end

\section{IV.CONCLUSION}

The proposed routing algorithm will help in routing the data packet through different nodes of the VANET network. As the most of the nodes of VANET is dynamic in nature so to find a better route is difficult and also may be time consuming.

So this proposed algorithm will exhibit some sort of intelligence in choosing the next node so that it can find out not the best but the better route to transmit the data packet.

Here any kind of existing shortest route algorithm is not used but to calculate the shortest route from the other routes, a new concept is used which is very easy to implement and also easy to understand. The proposed algorithm is coded in $\mathrm{C}$ language and the implementation takes round about 260 lines of codes including some comment lines. So the size of the 
code is not too big, it takes only $6 \mathrm{~KB}$ of memory. So for this flexible size of the code this can be easily implemented in the router which will release the pressure from the router.

As the nodes in VANET are uncertain so the distances are calculated using genetic algorithm where it tests all the possible nodes with a certain range of latitude and longitude and converges until the goal is reached.

The particular topics on which the work is done is not limited to what has been done through nearly more than 9 or 10 months during the project but it is more than this. Throughout the project work the main focus is to propose an algorithm and also the implementation of the algorithm through a programming language so that it can be proved easily that the algorithm is working properly. But in spite of all these due to limited time the following things should be incorporated in future scope.

- This has been implemented through C language but in future small models of cars should be built which can be move with the Wi-Fi set up inbuilt with it in which it can be shown that the message is transferring from one car to another car easily.

- Secondly, focus will be to better the heuristic by taking into consideration other networking parameters such as load in a node, delay threshold if any in the node etc.

- Thirdly, as the routing algorithm is intelligent so it is able to encrypt and decrypt the messages to be sent. Try to better the encryption technique in future so that it will be little bit of difficult for the intruders to decrypt the message.

\section{REFERENCES}

[1] Wireless ad hoc network From Wikipedia, the free encyclopedia

[2] Aarti , Dr. S. S. Tyagi, "Study of MANET: Characteristics, Challenges, Application and Security Attacks", International Journal of Advanced Research in Computer Science and Software Engineering, Volume 3, Issue 5, May 2013 ISSN: 2277 128X.

[3] Venkatesh, A Indra, R Murali, "Routing Protocols for Vehicular Adhoc Networks (VANETs): A Review" Journal of Emerging Trends in Computing and Information Sciences, Vol. 5, No. 1 January 2014 ISSN 2079-8407.

[4] Hiren Kathiriya , Nidhi Kathiriya and Arjav Bavarva, "Review on V2R Communication in VANET", Proceedings of International Conference on Innovations in Automation and Mechatronics Engineering 2013 (ICIAME-2013), 21-23 February 2013.

[5] Uma Nagaraj, Dr. M. U. Kharat, Poonam Dhamal, "Study of Various Routing Protocols in VANET", IJCST Vol. 2, Iss ue 4, Oct . Dec. 2011

[6] Manju Rani, Dr. Nasib Singh Gill, "Comparative Study Of Various Vanet Routing Protocols”, IJCSMS International Journal of Computer Science \& Management Studies, Special Issue of Vol. 12, June 2012.

[7] Sotirios Tsiachris, Georgios Koltsidas, Fotini-Niovi Pavlidou "Junction-Based Geographic Routing Algorithm for Vehicular Ad-hoc Networks"

[8] Mr. Pawan Sharma, Prof. Angeeta Hirwe, "Implementation in Vehicular Communication with Various Approaches \& Techniques", International Journal of Scientific Engineering and Applied Science (IJSEAS) - Volume-2, Issue-3,March 2016 ISSN:2395-3470.

[9] Kashif Naseer Qureshi, Abdul Hanan Abdullah, "Topology Based Routing Protocols For Vanet And Their Comparison With Manet", Journal of Theoretical and Applied Information Technology 31st December 2013. Vol. 58 No.3.

[10] Calculate distance, bearing and more between Latitude/Longitude points - (http://www.movable-type.co.uk/scripts/latlong.html) as on 27/03/17. (Reference helped in calculating the distance from latitude and longitude)

[11] Earth radius From Wikipedia, the free encyclopedia (Reference helped in calculating the distance from latitude and longitude)

[12] Arctangent information from - ( http:// www .icoachmath .com /math_dictionary / arctangent.html ) as on 9/05/17. 